\title{
Configurações
}

Revista de sociologia

\section{Revitalización urbana y especialización del centro histórico de Pontevedra}

Urban revitalization and tourist, trading and/or residential expertise

Revitalisation urbaine et spécialisation touristique, commerciale et/ou

résidentielle

\section{Sara María Torres Outón}

\section{OpenEdition}

\section{Journals}

Edición electrónica

URL: http://journals.openedition.org/configuracoes/1787

DOI: 10.4000/configuracoes. 1787

ISSN: 2182-7419

\section{Editor}

Centro de Investigação em Ciências Sociais

\section{Edición impresa}

Fecha de publicación: 1 junio 2013

Paginación: 129-141

ISBN: 1646-5075

ISSN: 1646-5075

\section{Referencia electrónica}

Sara María Torres Outón, « Revitalización urbana y especialización del centro histórico de Pontevedra », Configurações [En línea], 11 | 2013, Puesto en línea el 22 septiembre 2014, consultado el 01 mayo 2019. URL : http://journals.openedition.org/configuracoes/1787 ; DOI : 10.4000/configuracoes.1787

Este documento fue generado automáticamente el 1 mayo 2019. 


\title{
Revitalización urbana y especialización del centro histórico de Pontevedra
}

\author{
Urban revitalization and tourist, trading and/or residential expertise \\ Revitalisation urbaine et spécialisation touristique, commerciale et/ou \\ résidentielle
}

Sara María Torres Outón

\section{Introducción}

1 Aunque los procesos de revitalización urbana suelen identificarse con un gran proyecto o icono de la transformación del espacio, lo determinante son las estrategias, gestiones y decisiones que se desarrollan para impulsarlo y la contribución de los diferentes agentes sociales, económicos y políticos para conseguirlo. En esta ocasión, se analiza el proceso de transformación del centro histórico de Pontevedra a partir de una problemática común a los centros históricos y una solución local impulsada, en primer lugar, por los comerciantes. De forma voluntarista y sin contar con una estructura jurídica, los comerciantes iniciaron acciones para frenar el deterioro espacial de su entorno. Crearon en 1989 la Asociación Cultural Zona Monumental al objeto de promover el casco histórico como identidad cultural de la ciudad. Ésta se transformó en el año 2003 en Centro Comercial Urbano Zona Monumental, carácter más profesional pero continuadora de la apuesta turística- cultural.

2 Para comprender esta estrategia de desarrollo vinculada al territorio es necesario conocer tanto la trayectoria de los comerciantes como los procesos de regeneración urbana impulsados desde la Administración. Por ello, junto con la revisión documental sobre cascos históricos, turismo y comercio, hubo que consultar información de la asociación (memoria de actividades desde su creación, informes internos, etc), del Plan Urban Pontevedra 1996-1999 y prensa. Así mismo, se realizaron tres entrevistas en 
profundidad a miembros de la directiva (al Presidente y Vicepresidente al inicio de transformación -mayo 2001 y con la creación de Centro Comercial Urbano -noviembre 2003- y, al Presidente entrante y saliente con el cambio de presidencia -junio 2008).

3 Aunque se abordan problemas comunes resulta interesante conocer cuáles han sido las soluciones locales, aquellas capaces de poner en valor la unicidad del espacio y siempre guiadas por principios económicos, rendimiento social, viabilidad medioambiental y corrección técnica (Coca-Stefaniak, 2009: 74, 79).

\section{Cascos históricos: Evolución y Especialización}

4 Esta investigación entronca con temáticas fuertemente interrelacionadas entre sí: 1) la degradación de los cascos históricos; 2) su especialización, bien sean comercial, turística y/o residencial; y 3) las estrategias para su revitalización.

5 Inicialmente han surgido iniciativas orientadas a la rehabilitación de edificios y recuperación de espacios (transformación física/renovación urbana) y, posteriormente, otras más globales que pretenden incidir no sólo sobre aspectos arquitectónicos o patrimoniales sino sociales y económicos (transformación física y funcional/ revitalización urbana). Estas últimas persiguen la puesta en valor del espacio, no sólo a través de la reorganización funcional sino socioeconómica, de manera que se solventen las causas (condiciones económicas, físicas, sociales y ambientales) que generan los principales problemas urbanos

6 El comercio, actividad económica tradicional de muchos cascos históricos, pugna por mantenerse como motor de aquéllos con especialización comercial (comercio minorista local) o transformarse análogamente con la especialización turística (comercio para turistas). En ambos casos, es preciso establecer alianzas con las nuevas actividades que se han ido asentado en estos espacios ligada a la hostelería, la administración o el ocio. Pese a todo, los centros históricos, más allá de su valor patrimonial o económico son espacios vivos y vividos; por eso, el uso residencial ha de predominar sobre todos ellos; puesto que es su uso natural.

\subsection{Cascos históricos: abandono y degradación}

7 El proceso de deterioro de los cascos histórico se remonta a la revolución industrial. En una primera fase (1850-1950), fruto del crecimiento demográfico e inmigración rural, los cascos sufrieron una notable densificación que derivó en congestión e, incluso, hacinamiento. La posguerra (década de los 50-70) dio paso a una segunda fase en la que se invirtió el proceso. Para acoger a la nueva población, surgieron los ensanches a modo de nuevos polígonos residenciales sustrayendo de la función residencial a los centros históricos. El crecimiento de la ciudad y aparición de nuevos ensanches para uso residencial fueron los motivos principales del abandono de viviendas y despoblación, que propició el desarrollo de un proceso creciente de abandono, degradación y marginalización de los cascos históricos. Los edificios de los cascos históricos se quedaban desocupados o con inquilinos en régimen de alquiler de rentas antiguas, lo que hacía inviable invertir en su conservación y mejora. Comienza así un círculo de deterioro de los centros históricos que no se limita a la cuestión urbanística (derrumbe de edifi cios) sino que va acompañado de una problemática social de gran calado: marginalidad, deterioro 
de la calidad de vida, del medio ambiente urbano, etc. En la década de 70-80 arrancó, no de manera uniforme, la revalorización del precio del suelo al recuperar los cascos históricos su centralidad, bien por ser objeto de deseo de las clases dominantes, bien por su aprovechamiento terciario (Campesino, 1984: 53-59). A partir de entonces, surgen un interés y preocupación por recuperar los cascos históricos, y diversas estrategias, con diferente grado de éxito, para conseguir su revitalización funcional y desarrollo sostenible (López López, A., 2005: 332). España cuenta con cascos urbanos con gran valor artístico-patrimonial y monumental gracias a la protección e inversión de la administración (Bosque Maurel, J., 2011: 20), si bien resulta necesario el apoyo de otros agentes.

8 En definitiva, los cascos históricos de nuestro entorno independientemente de su funcionalidad (centralidad histórica y cultural, funcional, mixta o pérdida de ésta) o desarrollo urbano (concentración vs desconcentración) se caracterizan, en mayor o menor grado, por haber sufrido problemas de despoblamiento y abandono de la vida urbana y, consecuentemente, cambio de usos. En primer lugar, el crecimiento de las ciudades y el traslado de población derivaron en una pérdida sustancial de la especialización residencial. En segundo lugar, la pérdida de población y los cambios en los hábitos de consumo afectó a la pérdida del uso comercial que ha ido trasladándose al ensanche y a la periferia (centros comerciales cerrados). Ambas situaciones han supuesto un abandono de usos tradicionales (residencial y comercial) y la aparición de actividades nocturnas incompatibles con el uso residencial y/o una creciente terciarización ligada a actividades administrativas, turísticas y/o lúdicas y, en algunos casos, proliferación de comportamientos incívicos (Santamaría Camallonga, J., 2013: 119-122).

\subsection{Especialización de los centros históricos}

\section{Comercial}

9 Los cascos históricos, en la medida en que ocupan un lugar central en la ciudad, han tendido a acoger en su interior una actividad comercial, compatible con el uso residencial, que reforzaba esta centralidad. A la problemática propia de los centros históricos es necesario añadir los efectos y consecuencias que sobre la actividad comercial han tenido la aparición de centros comerciales periféricos y los cambios en lo hábitos de consumo que, indudablemente, han contribuido al descenso en el flujo de actividad en las pequeñas y medianas empresas ubicadas en la parte antigua de las ciudades.

10 La elevación de la renta per cápita y del poder adquisitivo de la población española que acompañaron al despegue económico de los años sesenta es uno de los principales factores explicativos de los cambios en los hábitos de compra.

11 Algunas de las características generales de este periodo son la generalización del automóvil como forma de desplazarse hacia el punto de consumo, incorporación masiva de la mujer al trabajo, cambios en la estructura de la unidad familiar y en el equipamiento de la vivienda. Todo esto, en definitiva, conduce a un uso intensivo del tiempo, fuera de la jornada laboral convencional, que se retrae del acto de la compra para disfrutarlo conjuntamente con el tiempo dedicado al disfrute de la vida familiar y el ocio (Pérez Vilariño, 2002: 30).

12 Esta reducción de comercios, que constituían un pilar económico y motor de gran importancia, ha reforzado la marginación y abandono de los centros históricos. A esta 
redistribución de los flujos comerciales internos de la urbe hay que sumar otros factores que explicarían las dificultades del comercio para paralizar la pérdida de especialización comercial de los cascos históricos. Se trata de características relativas a los propios establecimientos: disponen de menos recursos (humanos y materiales), suelen ser pequeñas empresas, con escasa o insufi ciente flexibilidad para adaptarse a las demandas de los ciudadanos, reducidas dimensiones, con frecuencia negocios familiares, menor disponibilidad de información para hacer frente a los avatares del mercado de consumo, menor capacidad para negociar créditos y ayudas, etc (Molinillo Jiménez, 2002: 27-8; Rovira Lara, 2012: 20, Pérez Vilariño, 2002: 32). En definitiva, tienen más dificultades a la hora de competir en un mercado en continuo cambio.

Por tanto, los cascos históricos con especialización comercial han tenido que enfrentarse a dos problemáticas: una derivada de su condición de espacio cuyo medio ambiente urbano se ha visto deteriorado por el abandono de la actividad residencial; y, otra, el descenso de la actividad económica comercial. Por ello, en los últimos treinta años ha surgido a nivel local una figura clave en el impulso y gestión de los centros históricos: las asociaciones profesionales de comerciantes y los gerentes de centros comerciales urbanos; quienes han desempeñado un papel fundamental en la animación, dinamización y revitalización de estos espacios (Coca-Stefaniak y otros, 2009: 74-75).

\section{Turística}

14 No cabe duda de la trascendencia económica, social y cultural del turismo, si bien como actividad económica transversal resulta ciertamente imprecisa ya que engloba un gran abanico de subsectores productivos que no siempre son claramente identificados (Orgaz Agüera, F., 2013: 3). Favorece la creación de negocios (comercio y hostelería en mayor medida) y estimula inversiones en infraestructuras y mejoras que redundan en el bienestar de la población residente.

Si bien, precisa de unos requisitos mínimos relativos a los servicios y equipamientos básicos para el visitante relacionados tanto con la oferta de alojamiento y restauración, transporte, comercio, información turística, actividades culturales como con los propios recursos patrimoniales y culturales (Dal Molin, E. D y otros, 2008: 8). E indudablemente tiene unos límites derivados de su capacidad de acogida, tanto en relación a las dimensiones cuantificables (ecológica, física y económica) como a las interrelaciones generadas y a la acción política (López López, A., 2005: 340).

16 El potencial turístico de los centros históricos no tiene porque reducirse a la visita de monumentos o al valor patrimonial del espacio sino que incluye otras categorías que enlazan con las expresiones culturales, artísticas y/o creativas que se en el espacio, las funciones y la vida social (Fernández Salinas, 1999:19; 2008:3,4; González González, M. J., 2005: 369-371; Ruíz Baudrihaye, J. 1997: 46-48; Zallo, R., 2009: 3):

17 - Territorio, entendido en el sentido más amplio del término, es decir, patrimonio, bienes culturales, trama urbana y lógica espacial.

18 - Vida urbana producida por las funciones y actividades que acoja, sus usos (tradicionales y/o actuales) y los equipamientos existentes (comercios, negocios y equipamiento institucional);

19 • Sociabilidad generada como imaginario social, lugar de encuentro y seña de identidad. 
20 A la luz de los recursos disponibles en los cascos históricos parece que el aprovechamiento turístico de tipo cultural es una alternativa viable. No sólo constituye la base para la puesta en marcha de actividades económicas ligadas a la venta de productos (no sólo comercio turístico sino comercio minorista en general) y consumo (hostelería, restauración, ocio); sino que posee un efecto multiplicador que lo convierte en todo un revulsivo para la recuperación urbana (Troitiño Vinuesa y otros, 1998b: 313), sin caer en el error de considerar -como ha ocurrido en algunos casos por parte de la administración - que esta función por sí sola es el recambio a las actividades en detrimento (Troitiño Vinuesa, 2009: 150 y 177).

21 Las sinergias entre el turismo cultural y el comercio en los cascos históricos favorece la conservación del patrimonio histórico y la recuperación de espacios culturales públicos como plazas y de consumo cultural bien sea privados (restaurantes y tiendas a pié de calle) o públicos (Torres Outón, 2013: 14). Si bien, ha de hacerse de forma controlada de modo que no derive en problemas de congestión, encarecimiento del coste de la vida para los residentes, especulación, fuga tanto de residentes como de actividades no turísticas, y banalización social económica y cultural de la comunidad de acogida; en definitiva, compatibilizar el uso turístico con la multifuncionalidad de estos espacios (Troitiño Vinuesa y otros, 1998a: 82-91; 2009: 151).

\section{Residencial}

Para comprender el proceso de regeneración de los cascos históricos iniciado en los noventa es necesario señalar que: 1) "lo que más ha contribuido a la conservación de muchos de estos conjuntos ha sido el escaso interés económico de estas zonas por estar situadas dentro de posiciones marginales en la división territorial del trabajo" (Santamaría Camallonga, J., 2013: 127); 2) fue posible acometer proyectos con orientación multidimensional, enfoque territorial integrado y participación ciudadana a través de la financiación comunitaria (Dreve, $p, 2008: 183$ ).

El Plan Urban permitió poner en marcha proyectos ligados al desarrollo económico, la mejora de las condiciones de vida de los residentes de la zona y la incorporación de estos espacios a la dinámica social y económica de la ciudad (Valenzuela Rubio, 2000: 94). Por un lado, impulsó la recuperación de espacios públicos y edificios señeros por ser éstos vestigios claves del patrimonio histórico y arquitectónico, muestra de su centralidad, depositarias de las memoria social y preservación de la memoria urbana y colectiva (Gutmann, C. S., \& Schicchi, M. C., 2013: 18). La rehabilitación de viviendas -ante la escasa participación pública- dependió de la iniciativa privada, aquejada bien de dificultades financieras de los propietarios como de falta de concienciación y sensibilidad (Tomé Fernández, 2007: 78).

Ni la financiación comunitaria, ni la ubicación de servicios públicos, ni las estrategias comerciales y turísticas -que abogaban por la recuperación de los cascos y su revitalización a través de la atracción de visitantes y turistas con la actividad comercial y terciaria- han conseguido mejoras significativas respecto al uso residencial. "Normalizar los espacios urbanos históricamente configurados significa devolverles la condición de lugares diversos, económica y socialmente" (Tomé Fernández, 2007: 84), y ese objetivo no se ha alcanzado pese a los logros conseguidos y a la recuperación y revitalización de muchos de los centros históricos españoles. La atracción y uso residencial, en lugar de 
normalizarse, ha dado lugar a fenómenos de especialización social ligados a clases altas (gentrificación), extranjeros (marginalización) o estudiantes.

\section{Zona monumental de Pontevedra. Un caso de estúdio}

\subsection{Punto de partida} En el caso objeto de esta investigación, el punto de partida se sitúa en el proceso de degradación y abandono que todavía estaba presente en el casco histórico de Pontevedra españolas, en Pontevedra el crecimiento de la ciudad tradicional y aparición del nuevo ensanche implicó el abandono de las funciones tradicionales: uso residencial y comercial. El deterioro físico y decadencia social transformaron este espacio, en su día centro y seña de identidad de la ciudad. Al inicio de los noventa, la Zona Monumental pontevedresa concentraba una problemática demográfica, socioeconómica y urbana. Este espacio se caracterizaba por (Valenzuela Rubio, 2000: 98; Pérez Vilariño, 2002: 12; Torres Outón, 1994: 140; padrón de habitantes 1991):

- Abandono de viviendas (35\% de las viviendas están desocupadas)

- Envejecimiento de la población (18\% es mayor de 65 años).

- Alta tasa de paro (20\%).

- Mayor índice de vivienda en alquiler (menos del 70\% de los residentes son propietarios).

1 - Nivel bajo de instrucción (16\% sin estudios o analfabeta).

- Bajos niveles de renta (3,2\% población receptora de subsidios o por debajo del SMI)

- Obsolescencia de infraestructuras urbanas.

- Escasez de equipamientos urbanos.

- Pérdida de atracción comercial y declive de las actividades económicas.

- Drogadicción.

- Delincuencia e inseguridad ciudadana.

Esta caracterización le permitió participar de las ayudas del Plan Urban en el periodo 1994-1999 para acometer una transformación del espacio sin precedentes, especialmente por la recuperación de espacios públicos (plazas), rehabilitación de edificios claves 
(Mercado de Abastos) y la peatonalización. En relación a la iniciativa privada, en este caso, la gestión de ayudas -aún siendo de limitada cuantía- y la concienciación devino en obras de embellecimiento de fachadas y recuperación de rótulos comerciales.

\subsection{Algunos indicadores}

39 Respecto al uso residencial cabe decir que, a pesar de la revitalización acometida, no se ha frenado la pérdida de población. En el periodo 1999-2010, según los datos del padrón de habitantes, la población del municipio aumentaba en un 10,5\% mientras que el casco histórico perdía un 15,5\%, lo que supone que en éste reside sólo un 2,5\% del total. En este mismo periodo, la población extranjera creció, pasando de representar el 2,20\% de los residentes del casco histórico en el año 1999 a 7,47 en el año 2010 (casi un tercio proceden de Bangladesh y Brasil). A pesar de lo negativo de estos datos, "el centro histórico de Pontevedra es uno de los más habitados de Galicia, con una población de 1972 personas y unos 730 inmuebles" (Pérez, L., 2009).

40 En cuanto a la actividad económica, un análisis comparativo entre el año 2002 y 2012 sobre el uso de los locales comerciales del casco histórico (bajos) afirmaba que: 1) incrementó la actividad diurna con un crecimiento de más de 5,87\% de hostelería (cafeterías, taperías y restaurantes) seguida por comercios de complementos (2,35\%); 2) se mantiene la actividad nocturna de pubs y locales de copas; 3) se redujeron de forma importante los negocios dedicados a los servicios personales (peluquerías) y comercio de proximidad; y 4) creció-en torno a un 5\%- el número de locales vacíos (Brañas, P., 2012: 52-54). Con lo cual se aprecia el mantenimiento y crecimiento de actividades económicas ligadas a la hostelería diurna y comercio especializado al tiempo que se alerta sobre la caída de actividad en el comercio de barrio y desaparición de negocios.

41 Relativo a la asociación y según información obtenida en la entrevista de junio 2008, cabe señalar que el número de asociados aumentó progresivamente desde sus inicios -una decena- hasta el año 2007 en el que jubilaciones anticipadas, cierres de negocio y bajas por razones económicas ha supuesto un fuerte varapalo a la entidad. El mayor crecimiento se produjo en los años 2003-4 debido a la ampliación de nuevos sectores (hostelería y servicios) y a la difusión de la nueva entidad superando los ciento ochenta asociados, cerca del $80 \%$ del comercio asentado en el casco histórico y un $40 \%$ de la hostelería y profesionales. La transformación en Centro Comercial Urbano supuso una inyección de asociados más jóvenes, negocios más innovadores; en definitiva, una nueva generación.

\subsection{Acciones acometidas por los comerciantes}

42 El declive en la actividad comercial, por traslado del grueso de la actividad comercial del casco histórico -con larga tradición de comerciantes y mercaderes-al ensanche, acentuó su abandono y degradación. Posteriormente la competencia de dos grandes superficies instaladas en la periferia a finales de la década de los ochenta, la primera, y de los noventa, la segunda, dificultó más la labor comercial. Esta situación de pérdida de centralidad y decadencia hizo surgir el movimiento asociativo entre los comerciantes y descubrió como actor principal de desarrollo no sólo comercial sino turístico y culturalde la Zona Monumental. El germen asociativo, tal y como declaran desde la directiva, 
"no surgió sólo porque añorásemos la época dorada del comercio del casc antiguo sino porque queríamos frenar el deterioro que estábamos sufriendo. Tenemos aquí nuestros negocios, el local es nuestro..., muchos vivimos aquí o tenemos propiedades. Veíamos desaparecer nuestro mundo (...) En el año 88 empezamos a patrullar la noche para evitar la drogadicción y los robos, y proteger nuestros negocios (...), y así nacimos,.... como asociación cultural" (entrevista mayo 2001).

Para romper la inercia de abandono y devolver al espacio histórico su centralidad, organizaron las primeras visitas guiadas orientadas no tanto a turistas como a visitantes y residentes. Se trataba de conseguir que en el mapa mental de la población de la ciudad y el entorno se incorporase el centro histórico como un lugar más de la ciudad. Éste era el primer paso para lograr recuperar los valores identitarios del centro histórico y asociarlo con valores en alza (espacio público, corazón histórico, patrimonio, etc). El diseño, gestión y puesta en marcha de productos turísticos en el casco histórico de la ciudad de Pontevedra fue impulsado por los comerciantes -los agentes sociales y económicos que desarrollan su actividad en este espacio- y no por una entidad propiamente turística o ligada a la Administración. Éstos, conocedores de los recursos y potencial del espacio en el que desarrollan su actividad, apostaron por el turismo como vía para iniciar su recuperación, como punto de partida para su revitalización económica y social. La apuesta turística supuso un punto de inflexión en el proceso de abandono y degradación en el que estaba sumergido el casco histórico a finales de los ochenta. Los comerciantes se asociaron con el objetivo de promover actividades turísticas que introdujeran visitantes y turistas en el casco histórico, un primer paso para recuperarlo como espacio público y lugar de encuentro.

La alianza entre turismo y comercio deviene, en muchas ocasiones, en una actividad comercial enfocada básicamente al turismo. Sin embargo, en este caso, en la medida en que fue el comercio el que impulsó el turismo, esto no ha derivado en una especialización turística del comercio sino en un refuerzo del papel de la hostelería diurna, tanto para turistas como para residentes o visitantes, y el mantenimiento de un comercio minorista para la ciudadanía.

Posteriormente, la transformación urbana que supuso el Plan Urban ya mencionado, permitió acometer importantes obras, tales como, la mejora de servicios básicos para uso residencial (viviendas) y económico (negocios), la rehabilitación de edificios nobles (Casa da Luz, Pazo de Murgartegui, Casa Azul y remodelación del Mercado de Abastos) y regeneración del espacio urbano con la peatonalización y recuperación de espacios públicos (plazas).

"La peatonalización ha sido claramente positiva. Ha dado nuevos clientes, por un lado los de la propia ciudad que han incorporado ciertas plazas a su lugar de paseo habitual, como lo estaba siendo las calles del centro, si bien había que compensar la pérdida de aquellos que paraban al pasar en coche (...). En cuanto al tema de accesos, hay que dejar que concluyan las obras y después decidir a tres bandas: ayuntamiento, comercio y transportistas, qué hacer" (entrevista mayo 2001).

En este momento, la Asociación operó como mediador y contribuyó a solventar temores y oposiciones a las obras.

La programación cultural y actividades de animación realizada a través de la alianza con la cultura, el ocio y el turismo ha resultado válida para atraer población y dinamizar las plazas. Pero se hacía necesario establecer alianzas con otros sectores y entidades de la ciudad, por ello se iniciaron acuerdos de colaboración con regentes y asociaciones de hostelería o servicios. 
"Nos hemos profesionalizado porque ya no llega con campañas de animación de calle y dinamización (...). Es nuestra misión compatibilizar intereses, (...) ofrecer servicios tanto al comercio como a hostelería y profesionales que ejerce en el casco histórico. (...) Incluso negociar con el Ayuntamiento (...)" (entrevista noviembre 2003).

Por ello a las visitas guiadas siguieron otras actividades de menor alcance pero con más participación local. Unas se han consolidado y llevan ya diferentes ediciones (Fiesta de la calabaza y Feria de Otoño para exaltación de productos alimenticios de época, o Exposición de arte en los escaparates; ambas en colaboración con asociaciones locales), otras se mantienen pero ya no dependen de la asociación como las visitas guiadas (hoy en manos de la Administración), y otras no han progresado como la exposición obras de arte de Jóvenes Talentos (muestra de obras realizadas por estudiantes de la Facultad de Bellas Artes) que ha sido sustituida de forma espontánea por un mercadillo de antigüedades. "Hay que seguir trabajando, innovando, (...) renovarse o morir" (entrevista junio 2008).

En Pontevedra, los comerciantes asociados del Zona Monumental constituyen un actor local de reconocido prestigio, cuyas estrategias y acciones de desarrollo local tienen capacidad para iniciar procesos de cambio (Iglesias, 2013). Han sido parte activa de los grandes procesos acontecidos en el casco histórico, primero defendiendo su recuperación (1989), apoyando la reforma urbana (1999), abriendo la asociación a nuevos sectores de actividad (2003) y consolidándose como entidad empresarial local de reconocido prestigio (actualidad) que con presencia en la ciudad defienden la dinamización del centro histórico y la promoción comercial.

\section{Conclusiones}

50 A través de esta experiencia se ha podido comprobar, en primer lugar, que los actores sociales no sólo han de ser tenidos en cuenta en toda estrategia de desarrollo local sino que son una pieza fundamental para impulsar el cambio. La interacción e implicación de los comerciantes asociados con su espacio les ha permitido iniciar un proceso ilusionante de inclusión al que se han sumado: la administración como cofinanciadora de su programación cultural y turística, y actualmente gestora de las visitas guiadas; la hostelería vinculada a campañas comerciales y de animación de calle; y la ciudadanía al participar en las actividades.

51 Cabe destacar, en segundo lugar que, a pesar de que los comerciantes no son expertos en turismo, sienten y viven los recursos del espacio en el que generan su actividad y, por ello, han sido capaces de impulsar procesos de transformación a través de acciones concretas en las crean y legitiman productos turísticos (inicialmente visitas guiadas y posteriormente ruta de heráldico, por ejemplo). Esta primera iniciativa -las visitas guiadas- consiguieron hacer del casco histórico un espacio de referencia; ya que cuando se iniciaron las primeras acciones, éste se caracterizaba por un avanzado estado de deterioro físico y social (1989) y no existían otros agentes (ni administración, ni hosteleros, ni profesionales del turismo) cuya labor se dirigiese a su promoción o recuperación. Por último, es preciso reconocer la valiosa contribución de la reforma urbana impulsada por el Plan Urban a finales de los noventa y continuada por la Administración Local. Sin esta transformación física difícilmente hubiese sido posible impulsar otras dinámicas de recuperación, ni por parte de los comerciantes, ni de otros agentes. 

revitalización ha sido vital pero que la batalla sigue librándose: 1) se ha frenado el proceso de degradación y se ha transformado el espacio en términos de preservación histórica, consumo cultural y sociabilidad; 2) se han consolidado ciertos flujos y movimientos de personas que pasean, trabajan y compran en el casco histórico; pero, 3) la actividad económica se ha reducido en general y; 4) el uso residencial sigue decreciendo con procesos de sustitución de población local por foránea.

Hoy en día la Zona Monumental ha recuperado ser seña de identidad de la ciudad y lugar de sociabilidad. El casco histórico es hoy un espacio vivido en mayor medida que hace tres décadas aunque debe afrontar su normalización en términos de recuperación del uso residencial y mayor atracción comercial sobre los consumidores, ya que tiende a concentrar actividades hosteleras.

\section{BIBLIOGRAFÍA}

BOSQUE, Maurel, J. (2011), "El patrimonio natural e histórico-monumental español. Algunos problemas actuales", Cuadernos Geográficos, no 48: 9-45.

BRAÑAS, Vázquez, P. (2012), Especialización y usos del Centro Histórico de Pontevedra, Trabajo Fin de Grado, Grado en Dirección y Gestión Pública, Universidad de Vigo, mayo.

CAMPESINO, Fernández, A. J. (1984), "Los centros históricos. Análisis de su problemática”, Norba, Revista de Geografía, nำ 5: 51-61.

CAMPESINO, Fernández, A. J., Coord. (1999), Comercio, Turismo y Cambios Funcionales en las Ciudades Españolas Patrimonio de la Humanidad, Cámara Oficial de Comercio e Industria de Cáceres.

COCA-STEFANIAK, J. A.; PARKER, C.; QUIN, S.; RINALDI, R., \& BYROM, J. (2009), “Town centre management models: A European perspective", Cities, nํ 26 (2): 74-80.

DAL MOLIN, E. D.; DOS SANTOS, A. S. \& OLIVEIRA, J. P. (2008), "Paisagem Urbana e Uso Turístico: Revitalização da Rua Hercílio Luz em Itajaí (SC)”, Revista electrónica de turismo cultural, Volume 2 n.ำ 1, 1ํㅗㄹ semestre: 1-22.

DREWE, P. (2008), “The URBAN Initiative or the EU as Social Innovator?”, in Paul Drewe, JuanLuis Klein and Edgard Hulsbergen (eds.), The Challenge of Social Innovation in Urban Revitalization: 183-196, Techne Press ed.

FERNÁNDEZ SALINAS, V. M. (2008), “La protección del Patrimonio Mundial en España”, E-rph: Revista electrónica de Patrimonio Histórico, no 2: 36-66.

FERNÁNDEZ SALINAS, V. M. (1999), “Centros Históricos y Medio Ambiente Urbano: un Futuro para la Calidad Urbana a Partir de su Pasado", Vivir las ciudades históricas: Ciudad histórica y calidad urbana: Seminario, Burgos, 19, 20 y 21 de enero de 1998: 225-238.

GONZÁLEZ GONZÁLEZ, M. J. (2005), “El desarrollo económico sostenible de los centros históricos”, Ería. Revista cuatrimestral de Geografía, no 68: 365-372. 
GUTMANN, C. S., \& SCHICCHI, M. C. (2013), “O valor patrimonial dos espaços públicos no centro da cidade de Valinhos (SP)”, Arquitetura Revista, n.o 9 (1): 9-19.

IGLESIAS, J. (2013), “Antes de que surgiese la asociación, la zona era un lugar de aparcamiento y trapicheo", Diario de Pontevedra, publicado el 31 de mayo.

LAGE, L. (2011): “Pontevedra deja de ser lugar de paso para los turistas", Diario de Pontevedra, publicado el 1 de septiembre.

LÓPEZ LÓPEZ, Alejandro (2005), “Desarrollo sostenible: medioambiente y turismo en las ciudades históricas. El caso de Toledo”, Observatorio Medioambiental, nº 8: 331-344.

MOLINILLO JIMÉNEZ, S. (2002), Centros comerciales en áreas urbanas, ESIC.

ORGAZ AGÜERA, F. (2013), "El club de producto turístico como herramienta para el desarrollo socioeconómico en países subdesarrollados", Contribuciones a la Economía, junio 2013, disponible en www.eumed.net/ce/2013/club-producto.html .

IGE (1999-2010), Padrón Municipal de habitantes, datos de población, Pontevedra, datos municipales.

PÉREZ VILARIÑO, J. y otros (2002), Horizonte comercial del casco histórico I, edita Seminario de Estudios Socioeconómicos de Pontevedra Carlos Velasco.

PÉREZ, L. (2009), “Unas 2.000 encuestas aclararán el estado de las viviendas de la zona monumental", Diario de Pontevedra el 5 de febrero.

ROVIRA LARA, A. y otros (2012), Gestión innovadora de centros comerciales urbanos. Modelos y experiencias, Tea.

RUÍZ BAUDRIHAYE, Jaime Axel (1997), “El turismo cultural: luces y sombras”, Estudios turísticos, no 134: 43-54.

SANTAMARÍA CAMALLONGA, J. (2013), “Centros históricos: análisis y perspectivas desde la Geografía", GeoGraphos, vol. 4, no 37: 115-137.

TOMÉ FERNÁNDEZ, Sergio (2007), “Los centros históricos en las ciudades españolas”, Eiría, oำ 72: 75-88.

TORRES OUTÓN, Sara Mª (1994), “Área Sociales en la ciudad” en Pontevedra: geografía social de un espacio, Premio Especial Diputación de Pontevedra 1996, sin publicar.

TORRES OUTÓN, Sara M (2013), “Una estrategia de revitalización impulsada por los comerciantes: simbiosis turismo y comercio en el casco histórico de Pontevedra", Aposta Revista de Ciencias Sociales, $\mathrm{n}^{\circ}$ 56, enero, febrero, marzo: 1-22.

TROITIÑO VINUESA, M. (2003), “Ciudades históricas y turismo sostenible”, en Revista de Arqueología, Arte y Urbanismo, no 4: 93-108, UNED.

TROITIÑO VINUESA, M. Á. \& TORRALBA, L. T. (2009): “Turismo y patrimonio en Castilla y León: las ciudades patrimonio de la humanidad (Ávila, Salamanca y Segovia) como destinos turísticos de referencia", Polígonos. Revista de Geografía, nº 19: 145-178.

TROITIÑO VINUESA, M. et al. (1998a), “Ávila: turismo y realidad urbana”, Cuadernos de la Escuela Regional de Turismo de Castilla y León.

TROITIÑO VINUESA, M. et al. (1998b), “Toledo: problemática e implicaciones urbanas del turismo", Eiría, no 47: 299-325.

VALENZUELA RUBIO, Manuel (2000), “La regeneración de los cascos históricos en España”, III Jornadas de Geografía Urbana, Oportunidades de desarrollo sostenible para los conjuntos urbano históricos, Burgos, 18-21 de mayo del 1998. 
ZALLO, R. (2009), "Industrias culturales y ciudades creativas”, Actas II Jornadas Ciudades creativas. Industrias digitales y ciudades creativas, Barcelona: 1-26.

\section{RESÚMENES}

El objetivo de este trabajo es conocer en qué medida las estrategias emprendidas por el comercio y, en particular, los comerciantes no sólo pueden contribuir al desarrollo local y revitalización del espacio, sino que resultan significativas por cuanto producen mudanzas en las percepciones sobre el espacio, lo que provoca cambios en los usos y especialización territorial. El estudio longitudinal de la actividad de los comerciantes del casco histórico de Pontevedra desde que se asociaron en el año 1989 hasta la actualidad, hará posible conocer cómo ha tenido lugar uno de estos procesos, cómo ha surgido esta apuesta de desarrollo local y en qué medida ha favorecido y/o se ha visto reforzada por otras actuaciones de regeneración urbana local.

The aim of this work is to know to what extent the strategies undertaken by trade and, in particular, the tradesmen can not only contribute to the local development and revitalization of the area, but also significantly generate shifts in the perceptions of the area, causing changes in land use and territorial specialization. The longitudinal study of the tradesmen's activities in the historic centre of Pontevedra since their association in 1989 until the present permits the discovery of how one of these processes took place, how this position of local development has emerged and to what extent has it encouraged and/or has been reinforced through other actions of local urban regeneration.

Le but de ce travail est de savoir en quelle mesure les stratégies entreprises par le commerce et, en particulier, par les marchands, peuvent contribuer non seulement au développement local et à la revitalisation de l'espace, mais qu'elles se révèlent aussi significatives quant à la quantité des transformations qu'elles génèrent dans les perceptions de l'espace, ce qui provoque des changements dans les usages et la spécialisation territoriale. Une étude longitudinale de lractivité des marchands du casque historique de Pontevedra depuis qu'ils se sont associés en 1989 jusquà maintenant, permettra de savoir comment s'est déroulé l'un de ces procédés, comment ce pari de développement local a vu le jour et en quelle mesure il a avantagé et/ou s`est vu renforcé par d'autres actions de régénération urbaine locale.

\section{ÍNDICE}

Mots-clés: centres historiques, développement local, commerce, tourisme culturel, patrimoine historique

Palabras claves: centros históricos, desarrollo local, comercio, turismo cultural, patrimonio histórico

Keywords: historic centres, local development, trade, cultural tourism, historical heritage

\section{AUTOR}

\section{SARA MARÍA TORRES OUTÓN}

Universidad de Vigo

Saratorres@uvigo.es 